\title{
Robustness of Aggregation in Networked Dynamical Systems
}

\author{
Luca Scardovi and Naomi Ehrich Leonard
}

\begin{abstract}
In this paper we study robustness of aggregation in networks of coupled identical systems driven by possibly different external inputs. This property is guaranteed by providing a finite $L_{2}$ gain condition for the closed-loop system. We show, for a class of systems, how robustness depends on the connectivity of the underlying communication graph. Applications range from coordination problems where there are conflicting objectives to the study of aggregation phenomena where perturbations of the nominal systems must be taken into account. Both scenarios arise in networks of biological and engineered coordinating systems.
\end{abstract}

\section{INTRODUCTION}

Aggregation of individuals is observed in biological systems at scales that range from cells to organisms. Aggregation plays a critical role in a variety of behaviors; for example, individuals gather to reproduce, forage and explore new regions [1], [2], [3]. Similarly, in engineered muti-agent systems, aggregation of agents is desirable for a variety of tasks. For example, in mobile sensor networks, aggregation enables communication and collective sensing activities such as coordinated gradient climbing and coverage for mapping or monitoring an uncertain environment [4], [5]. In all these scenarios, different individuals in the group may be subject to different external inputs. Heterogeneity in input signals could arise from perturbed or conflicting objectives across the group. For example, animals in a migrating group may have heterogeneous prior information on the location of the migration site, or a group of robots may receive noisy signals from the environment that drives their mission. For successful collective behaviors, aggregation should be robust to input heterogeneity.

In the present paper we study the robustness of aggregation of networked systems with respect to input heterogeneity. We measure aggregation by the dispersion of the individuals in the group: the smaller the dispersion, the higher the aggregation.

We formalize robustness with an $L_{2}$ gain condition for the networked system, and we derive the dependence of the $L_{2}$ gain on the communication topology. In particular we show, for a class nonlinear systems, that if the communication topology satisfies certain connectedness conditions, the dispersion of the group remains bounded in the presence of external disturbances with bounded $L_{2}$ norms. Furthermore, we provide an estimate of the dispersion as a function of the dynamical properties of the isolated systems and of the communication network.

L. Scardovi and N. E. Leonard are with the Department of Mechanical and Aerospace Engineering, Princeton University, USA. scardovi@princeton.edu, naomi@princeton.edu The work is supported by ONR grants N00014-02-1-0826 and N00014-04-1-0534.
In the first part of the paper, we prove the main result in the setting where each system is described by an inputoutput model. This mathematical model takes the form of an operator equation expressing the relationship between the inputs and the outputs. The relationship between the inputs and the outputs can be derived through experiments; this approach is therefore useful whenever the physical laws governing the system are absent or incomplete. The operator describing the systems is assumed to satisfy an incremental condition called relaxed co-coercivity [6]. This notion is the operator counterpart of the incremental feedback passivity condition proposed in a state-space setting in [7] to analyze synchronization of coupled oscillators.

In the second part of the paper we relate the obtained results to the case where each system is modeled with an "internal description" based on a state-space model.

As a special case of our analysis, we obtain a robustness result for consensus algorithms, which have received much attention in recent years [8], [9]. Robustness analysis of multi-agent networks has been considered in [10], in the case of leader-follower consensus.

The paper is organized as follows. In Section II notations are summarized and relevant operator concepts are reviewed. In Section III the model is introduced and the main result is stated. In Section IV we extend the obtained result to statespace models and, in Section $\mathrm{V}$, the particular case of linear systems is considered. Finally, in Section VI, we illustrate the theory with two examples.

\section{PREliminaries AND NOTATION}

\section{A. Notation and Terminology}

Throughout the paper we will use the following notation. Given $N$ vectors $x_{1}, x_{2}, \ldots, x_{N}$ we indicate with $x$ the stacking of the vectors, i.e., $x=\left[x_{1}^{T}, x_{2}^{T}, \ldots, x_{N}^{T}\right]^{T}$ and by $\bar{x}$ the average vector, i.e., $\bar{x}=\frac{1}{N} \sum_{i=1}^{N} x_{i}$. We denote with $I_{N}$ the diagonal matrix of dimension $N \times N$ and we define $1_{N} \triangleq[1,1, \ldots, 1]^{T} \in \mathbb{R}^{N}$. Given two matrices $A$ and $B$ we denote their Kronecker product with $A \otimes B$. For notational convenience, we use the convention $\hat{A}_{N}=A \otimes I_{N}$.

\section{B. Communication Graphs}

Given a set of interconnected systems the communication topology is encoded through a communication graph. The convention is that system $j$ receives information from system $i$ if and only if there is a directed link from node $j$ to node $i$ in the communication graph. Let $\mathcal{G}=\left(\mathcal{V}, \mathcal{E}, A_{d}\right)$ be a weighted digraph (directed graph) where $\mathcal{V}=\left\{v_{1}, \ldots, v_{N}\right\}$ is the set of nodes, $\mathcal{E} \subseteq \mathcal{V} \times \mathcal{V}$ is the set of edges, and $A_{d}$ is a weighted adjacency matrix with nonnegative elements $a_{k j}$. We assume 
that $\left\{v_{k}, v_{j}\right\} \in \mathcal{E}$ if and only if $a_{k j} \geq \eta, \eta>0$. The set of neighbors of node $v_{k}$ is denoted by $\mathcal{N}_{k} \triangleq\left\{v_{j} \in \mathcal{V}\right.$ : $\left.a_{k j} \geq \eta\right\}$. A path is a sequence of vertices such that for each of its vertices $v_{k}$, the next vertex in the sequence is a neighbor of $v_{k}$. We assume that there are no self-cycles, i.e., $a_{k k}=0, k=1,2, \ldots, N$.

The Laplacian matrix $L$ associated to the graph $\mathcal{G}$ is defined as

$$
l_{k j}= \begin{cases}\sum_{i=1}^{N} a_{k i}, & j=k \\ -a_{k j}, & j \neq k .\end{cases}
$$

The in-degree (respectively out-degree) of node $v_{k}$ is defined as $d_{k}^{i n}=\sum_{j=1}^{N} a_{k j}$ (respectively $d_{k}^{\text {out }}=\sum_{j=1}^{N} a_{j k}$ ). The digraph $\mathcal{G}$ is said to be balanced if the in-degree and the out-degree of each node are equal, that is,

$$
\sum_{j=1}^{N} a_{k j}=\sum_{j=1}^{N} a_{j k}, \quad k=1, \ldots, N .
$$

Balanced graphs have the particular property that the symmetric part of their Laplacian matrix is nonnegative: $L+$ $L^{T} \geq 0$ [11]. The following definition characterizes the concept of graph connectivity.

Definition 1: We say that the digraph $\mathcal{G}$ is connected if there exists a node $v_{k}$ such that all the other nodes of the graph are connected to $v_{k}$ via a path that follows the direction of the edges of the digraph.

Well known results from algebraic graph theory relate the connectivity of directed graphs to the spectral properties of the Laplacian matrix (or its symmetric part) [12]. In particular we define

$$
\lambda=\frac{1}{2} \lambda_{\min }\left[Q\left(L+L^{T}\right) Q^{T}\right]
$$

where $\lambda_{\min }$ denotes the minimum eigenvalue and where $Q$ is an $(N-1) \times N$ matrix such that $Q 1_{N}=0, Q Q^{T}=I_{N-1}$ and $Q^{T} Q=I_{N}-\frac{1}{N} 1_{N}^{T} 1_{N}$. The scalar $\lambda$ is called algebraic connectivity [12]. The algebraic connectivity is intimately related to the connectivity properties of the underlying graph and will allow for interesting graph theoretical interpretations of the results presented in the paper (see Remark 1).

\section{Operators}

We denote by $L_{2 e}^{m}$ the extended space of signals $v$ : $[0, \infty) \rightarrow \mathbb{R}^{m}$ which have the property that each restriction $v_{T}=\left.v\right|_{[0, T]}$ is in $L_{2}^{m}(0, T)$, for every $T>0$. Given an element $v \in L_{2 e}^{m}$ and any fixed $T>0$, we write $\|v\|_{T}$ for the $L_{2}^{m}$ norm of the restriction $v_{T}$, and given two functions $v, y \in L_{2 e}^{m}$ and any fixed $T>0$, the inner product of $v_{T}$ and $y_{T}$ is denoted by $\langle v, y\rangle_{T}$. We recall now some definitions about operators (the interested reader is referred to [13] and [6] for more details).

Definition 2: Let $H: L_{2 e}^{m} \rightarrow L_{2 e}^{m}$. Then $H$ is positive if

$$
\langle H u, u\rangle_{T} \geq 0, \quad \forall u \in L_{2 e}^{m}, \quad \forall T \geq 0 .
$$

Definition 3: Let $H: L_{2 e}^{m} \rightarrow L_{2 e}^{m}$. Then $H$ is relaxed co-coercive if $\exists$ some $\gamma$ such that for every pair of inputs $u, v \in L_{2 e}^{m}$

$$
\langle H u-H v, u-v\rangle_{T} \geq \gamma\|H u-H v\|_{T}^{2}, \quad \forall T \geq 0 .
$$

If $\gamma \geq 0$ then $H$ is called monotone. If $\gamma>0$ then $H$ is called co-coercive.

Clearly a co-coercive operator is monotone and relaxed co-coercive.

\section{OUTPUT AGGREGATION IN NETWORKS OF INPUT-OUTPUT SYSTEMS}

Consider $N$ identical systems, each characterized by an input-output operator

$$
H: L_{2 e}^{m} \rightarrow L_{2 e}^{m}: v_{k} \rightarrow y_{k}, \quad k=1, \ldots, N .
$$

We are interested in the analysis of the system

$$
y_{k}=H v_{k}, \quad k=1, \ldots, N,
$$

with input given by

$$
v_{k}=u_{k}+\sum_{j=1}^{N} a_{k j}\left(y_{j}-y_{k}\right), \quad k=1, \ldots, N
$$

where $u_{k} \in L_{2 e}^{m}$ are external inputs and the scalars $a_{k j}, k, j=1,2 \ldots, N$ are the coefficients of the adjacency matrix associated to the communication graph that couples the systems. For the sake of compactness we can rewrite the feedback law (2) as

$$
v(t)=u(t)-\hat{L}_{m} y(t) .
$$

We next formalize robustness of aggregation. We start by characterizing mathematically what aggregation means. Let $\Pi=I-\frac{1}{N} 1_{N} 1_{N}^{T}=Q^{T} Q$. Suppose that $m=1$ and observe that $\tilde{y} \triangleq Q y$ is equal to zero if and only if $y=\alpha 1_{N}$ for some $\alpha \geq 0$. It is evident then that $\|\tilde{y}\|_{T}^{2}$ is a measure of synchrony for the outputs and therefore will be called the output dispersion. When $m>1$ the concept is easily generalized defining $\tilde{y} \triangleq \hat{Q}_{m} y$. A low dispersion is associated to a high level of aggregation for the network.

Since we are interested in the analysis of the aggregation level, we define the operator $\tilde{H}: L_{2 e}^{m(N-1)} \rightarrow L_{2 e}^{m(N-1)}$ (associated to the closed-loop system (1) with the control defined by (2)) that acts on the transformed variable $\tilde{u} \triangleq$ $\hat{Q}_{m} u$ and produces the transformed output $\tilde{y}$. Intuitively, to be robust, a network must have a low output dispersion for reasonable values of the disturbances, i.e., the input dispersion $\tilde{u}$. This property can be guaranteed by requiring the operator $\tilde{H}$ to be finite-gain $L_{2 e}^{m(N-1)}$ stable, i.e., by requiring that there exists $\beta>0$ such that

$$
\|\tilde{y}\|_{T} \leq \beta\|\tilde{u}\|_{T}
$$

for every $u_{k} \in L_{2 e}^{m}, k=1,2, \ldots, N$ and every $T \geq 0$. The role of the interconnection term $\hat{L}_{m}$ in (3) is then to ensure that the input-output map $\tilde{H}$ has a finite $L_{2 e}^{m(N-1)}$ gain and, if possible, to minimize the $L_{2 e}^{m(N-1)}$ gain thus producing a 
robust networked system. In the following we exploit these ideas to analyze a class of nonlinear networked systems.

In Theorem 1 we prove that, if the operator $H$ is relaxed co-coercive, then for some classes of communication topologies, the input-output map $\tilde{H}$ is finite-gain $L_{2 e}^{m(N-1)}$ stable.

Theorem 1: Consider the closed-loop system defined by (1) and the inputs (3). Suppose that the operator $H$ is relaxed co-coercive. If $\lambda>-\gamma$, then for every $y$ and $u$ that satisfy (1) and (3) the following condition holds

$$
\|\tilde{y}\|_{T}^{2} \leq \tilde{\gamma}\langle\tilde{y}, \tilde{u}\rangle_{T}, \quad \tilde{\gamma}=\frac{1}{\gamma+\lambda}>0,
$$

for every $T \geq 0$, where $\tilde{u}=\hat{Q}_{m} u, \tilde{y}=\hat{Q}_{m} y$. As a direct corollary we also conclude that the operator $\tilde{H}$ is finite-gain $L_{2 e}^{m(N-1)}$ stable with $L_{2 e}^{m(N-1)}$ gain equal to $\tilde{\gamma}$, i.e.,

$$
\|\tilde{y}\|_{T} \leq \tilde{\gamma}\|\tilde{u}\|_{T},
$$

for every $u_{k} \in L_{2 e}^{m}, k=1,2, \ldots, N$.

Before proving Theorem 1 we introduce a preliminary Lemma.

Lemma 1: Let $v_{k}, y_{k}, k=1,2, \ldots, N$ be input-output pairs satisfying (1) and belonging to $L_{2 e}^{m}$. If the operator $H$ is relaxed co-coercive with coefficient $\gamma$, then

$$
\gamma\|\tilde{y}\|_{T}^{2} \leq\langle\tilde{y}, \tilde{v}\rangle_{T}
$$

holds for each $T \geq 0$, where $\tilde{y}=\hat{Q}_{m} y$ and $\tilde{v}=\hat{Q}_{m} v$.

Proof: Consider the scalar product

$$
\langle\tilde{v}, \tilde{y}\rangle_{T}
$$

and define $z_{k}=v_{k}-\gamma y_{k}$ that in vector form reads

$$
z=v-\gamma y \text {. }
$$

Define $\tilde{z}=\hat{Q}_{m} z$. By substituting (7) in (6) we obtain

$$
\langle\tilde{v}, \tilde{y}\rangle_{T}=\langle\tilde{z}, \tilde{y}\rangle_{T}+\gamma\langle\tilde{y}, \tilde{y}\rangle_{T} .
$$

Let's focus on the first term in (8):

$$
\langle\tilde{z}, \tilde{y}\rangle_{T}=\int_{0}^{T} z^{T}(t) \hat{Q}_{m}^{T} \hat{Q}_{m} y(t) d t .
$$

Since the operator $H$ is relaxed co-coercive, we have that

$$
\begin{aligned}
\left\langle z_{i}-z_{j}, y_{i}-y_{j}\right\rangle_{T}= & \left\langle v_{i}-v_{j}, y_{i}-y_{j}\right\rangle_{T} \\
& -\gamma\left\langle y_{i}-y_{j}, y_{i}-y_{j}\right\rangle_{T} \geq 0,
\end{aligned}
$$

for $i, j=1,2, \ldots, N$. By summing (10) over $i, j=$ $1,2, \ldots, N$ and by dividing by a normalization constant we get

$$
\frac{1}{2 N} \sum_{i, j=1}^{N}\left\langle z_{i}-z_{j}, y_{i}-y_{j}\right\rangle_{T}=\langle z, y\rangle_{T}-N\langle\bar{z}, \bar{y}\rangle_{T} \geq 0 .
$$

The right hand side in (11) is equal to (9). In fact,

$$
\begin{aligned}
\langle\tilde{z}, \tilde{y}\rangle_{T} & =\left\langle z, \hat{Q}^{T} \hat{Q} y\right\rangle_{T}=\left\langle z, y-1_{N} \bar{y}\right\rangle_{T} \\
& =\langle z, y\rangle_{T}-N\langle\bar{z}, \bar{y}\rangle_{T} \geq 0 .
\end{aligned}
$$

From (8) and (12) we conclude that

$$
\langle\tilde{v}, \tilde{y}\rangle_{T}=\langle\tilde{z}, \tilde{y}\rangle_{T}+\gamma\langle\tilde{y}, \tilde{y}\rangle_{T} \geq \gamma\|\tilde{y}\|_{T}^{2}
$$

which is the desired inequality (5).

We are now ready to prove Theorem 1 .

Proof of Theorem 1: Consider the inputs

$$
v(t)=u(t)-\hat{L}_{m} y(t),
$$

where $L$ is the Laplacian matrix encoding the coupling and $u(t)$ are external inputs. From Lemma 1 and substituting (13) in (5) we get

$$
\gamma\|\tilde{y}\|_{T}^{2} \leq\langle\tilde{y}, \tilde{u}\rangle_{T}-\left\langle\tilde{y}, \hat{Q}_{m} \hat{L}_{m} y\right\rangle_{T} .
$$

Note that $I_{N}-Q^{T} Q$ is a projection matrix onto the range space of $1_{N}$. Because $L 1_{N}=0$, it follows that $\hat{L}_{m}\left(I_{m N}-\right.$ $\left.\hat{Q}_{m}^{T} \hat{Q}_{m}\right) y=0$ and, thus,

$$
\hat{L}_{m} y=\hat{L}_{m} \hat{Q}_{m}^{T} \hat{Q}_{m} y=\hat{L}_{m} \hat{Q}_{m}^{T} \tilde{y} .
$$

Using (15) we observe that

$$
\begin{aligned}
\left\langle\tilde{y}, \hat{Q}_{m} \hat{L}_{m} y\right\rangle_{T} & =\frac{1}{2} \int_{0}^{T} \tilde{y}^{T}(t) \hat{Q}_{m}\left(\hat{L}_{m}+\hat{L}_{m}^{T}\right) \hat{Q}_{m}^{T} \tilde{y}(t) d t \\
& \geq \lambda \int_{0}^{T} \tilde{y}^{T}(t) \tilde{y}(t) d t=\lambda\|\tilde{y}\|_{T}^{2}, \quad \text { (16) }
\end{aligned}
$$

where $\lambda$ is the smallest eigenvalue of the symmetric part of the reduced Laplacian matrix $Q\left(L+L^{T}\right) Q^{T} / 2$. Combining (14) and (16) we obtain

$$
\gamma\|\tilde{y}\|_{T}^{2} \leq\langle\tilde{y}, \tilde{u}\rangle_{T}-\lambda\langle\tilde{y}, \tilde{y}\rangle_{T} .
$$

Using the assumption that $\lambda>-\gamma$ we conclude that

$$
\|\tilde{y}\|_{T}^{2} \leq \tilde{\gamma}\langle\tilde{y}, \tilde{u}\rangle_{T}
$$

where $\tilde{\gamma}=\frac{1}{\lambda+\gamma}$. Applying the Schwartz inequality we obtain

$$
\|\tilde{y}\|_{T}^{2} \leq \tilde{\gamma}\|\tilde{y}\|_{T}\|\tilde{u}\|_{T}
$$

and we conclude that

$$
\|\tilde{y}\|_{T} \leq \tilde{\gamma}\|\tilde{u}\|_{T}, \quad \forall T \geq 0 .
$$

From Theorem 1 we observe that, if the systems are characterized by a relaxed co-coercive operator and the algebraic connectivity is sufficiently large, the output dispersion of the closed-loop system is bounded for any input disturbances belonging to $L_{2 e}^{m}$. Moreover, since $\tilde{\gamma}=1 /(\gamma+\lambda)$, the bound decreases monotonically as the algebraic connectivity increases.

Remark 1: As mentioned in Section II, the algebraic connectivity is related to the topology of the communication graph associated to the network. In particular, it is known that if the graph is not connected in the sense of Definition 1 , then $\lambda \leq 0$ (see [12]). Accordingly, as an immediate corollary of Theorem 1, we obtain that if the operator $H$ is co-coercive with a negative $\gamma$, a necessary condition for the output dispersion of the closed-loop system to be bounded for any input disturbances belonging to $L_{2 e}^{m}$ is that the graph describing the communication topology be connected (in the sense of Definition 1). Similarly, we also point out that a sufficient condition for $\lambda>0$ is for the graph to be connected and balanced. 


\section{STATE SPACE FORMALISM}

Theorem 1 takes advantage of the input-output formalism to analyze robustness of aggregation without the need of an internal description of each individual system.

In the case we have (or we need) a state-space model, we can still use the results of the previous section by defining the input-output operator associated to the statespace description when the initial conditions of the systems are all equal and fixed (for convenience we will fix them to zero). To be more precise consider the systems

$$
\begin{aligned}
\dot{x}_{k} & =f\left(x_{k}, v_{k}\right) \\
y_{k} & =h\left(x_{k}\right)
\end{aligned} \quad k=1, \ldots, N
$$

where $x_{k}(0)=0$ for every $k, x_{k} \in \mathbb{R}^{n}$ and $v_{k}, y_{k} \in \mathbb{R}^{m}$. We can define the input-output operator $H: L_{2 e}^{m} \rightarrow L_{2 e}^{m}$ by substituting any input $v_{k} \in L_{2 e}^{m}$ in (17), solving the differential equation, and substituting the resulting statespace trajectory in $y_{k}=h\left(x_{k}\right)$ in order to obtain the output function $y_{k}$. If we assume that the operator $H$ is well defined, then the results of the previous section still apply.

The assumption that the initial states of the systems are set to zero can be removed if the closed-loop system (17) equipped with the control law (2) satisfies appropriate reachability conditions.

Theorem 2: Consider the system (17) with zero initial conditions and let $H: L_{2 e}^{m} \rightarrow L_{2 e}^{m}$ be the operator defined by substituting any input $v_{k} \in L_{2 e}^{m}$ in (17), solving the differential equation, and substituting the resulting statespace trajectory in $y_{k}=h\left(x_{k}\right)$ in order to obtain the output function $y_{k}$. Consider now the closed-loop system (17) with arbitrary initial conditions $x(0)=x^{0}$ and equipped with the control law (2). If $H$ is well-defined and relaxed co-coercive, $\lambda>-\gamma$ and the system is zero-reachable, then there exists a constant $\hat{\beta} \in \mathbb{R}$ such that the solutions of (17) satisfy

$$
\|\tilde{y}\|_{T} \leq \tilde{\gamma} \hat{\beta}+\tilde{\gamma}\langle\tilde{u}, \tilde{y}\rangle_{T},
$$

for every $T \geq 0$ and any input $u_{k} \in L_{2 e}^{m}$.

Proof: Consider system (17) where the initial conditions $x(0)=x^{0}$ are arbitrary and the input $v_{k}$ given by (3). Let $x_{k}(\cdot)$ be the corresponding solutions and $y_{k}(\cdot)$ be the outputs for every $k=1,2, \ldots, N$. Consider now system (17) with zero initial conditions. From zero-reachability, there exist inputs $v_{k}=w_{k}:\left[0, T^{*}\right] \rightarrow \mathbb{R}^{m}$ such that the solutions at time $T^{*}$ reach the states $x_{k}^{0}$ for every $k=1,2, \ldots, N$. Consider now the input

$$
s_{k}(t)=\left\{\begin{array}{rc}
w_{k} & t \in\left[0, T^{*}\right] \\
v_{k}\left(t-T^{*}\right) & t>T^{*}
\end{array}\right.
$$

and let $z_{k}(\cdot)$ be the solution with initial state $z_{k}(0)=0$ and input $s_{k}(t)$ defined in (19). From causality we observe that $z_{k}\left(T^{*}\right)=x_{k}(0)$ and therefore $z_{k}\left(t+T^{*}\right)=x_{k}(t), t \geq 0$.

Consider the outputs $q_{k}(\cdot)$ associated to the solutions $z_{k}(\cdot)$ (with zero initial conditions and inputs $s_{k}(t)$ ). Observe that each $s_{k}$ is the sum of two signals in $L_{2 e}^{m}$ and therefore it is also in $L_{2 e}^{m}$. From Lemma 1 we know that

$$
\gamma\|\tilde{q}\|_{T^{*}}^{2} \leq\langle\tilde{w}, \tilde{q}\rangle_{T^{*}} \triangleq \bar{\beta}
$$

Observe that

$$
\begin{aligned}
& \gamma \int_{0}^{T^{*}}\|\tilde{q}(t)\|^{2} d t+\gamma \int_{T^{*}}^{T+T^{*}}\|\tilde{q}(t)\|^{2} d t \\
& \leq \bar{\beta}+\int_{T^{*}}^{T+T^{*}} \tilde{s}^{T}(t) \tilde{q}(t) d t .
\end{aligned}
$$

Since $z_{k}\left(t+T^{*}\right)=x_{k}(t), t \geq 0$, we observe that $q_{k}(t+$ $\left.T^{*}\right)=y_{k}(t), t \geq 0$. Therefore from (20) we conclude

$$
\gamma\|\tilde{y}\|_{T}^{2} \leq \bar{\beta}-\gamma\|\tilde{q}\|_{T^{*}}^{2}+\int_{0}^{T} \tilde{v}^{T}(t) \tilde{y}(t) d t .
$$

Since $\lambda>-\gamma$, from Theorem 1 we conclude that

$$
\|\tilde{y}\|_{T}^{2} \leq \tilde{\gamma} \hat{\beta}+\tilde{\gamma}\langle\tilde{u}, \tilde{y}\rangle_{T},
$$

that is, (18) holds with

$$
\hat{\beta}=\bar{\beta}-\gamma\|\tilde{q}\|_{T^{*}}^{2} .
$$

It is important to note that the inequality (18) is different from (4) due to the presence of the bias term $\hat{\beta}$. However, we can still use (18) to provide a finite-gain result for the closed-loop system (where a bias term is included). In fact

$$
\begin{aligned}
\|\tilde{y}\|_{T}^{2} \leq & \tilde{\gamma} \hat{\beta}+\tilde{\gamma}\langle\tilde{u}, \tilde{y}\rangle_{T} \leq \tilde{\gamma} \hat{\beta}+\tilde{\gamma}\langle\tilde{u}, \tilde{y}\rangle_{T} \\
& +\frac{1}{2}\|\tilde{\gamma} \tilde{u}-\tilde{y}\|_{T}^{2} \\
& =\tilde{\gamma} \hat{\beta}+\frac{\tilde{\gamma}^{2}}{2}\|\tilde{u}\|_{T}^{2}+\frac{1}{2}\|\tilde{y}\|_{T}^{2}
\end{aligned}
$$

and we conclude that

$$
\|\tilde{y}\|_{T}^{2} \leq 2 \tilde{\gamma} \hat{\beta}+\tilde{\gamma}^{2}\|\tilde{u}\|_{T}^{2} .
$$

Relaxed co-coercivity and co-coercivity are the operator counterpart of the properties of output feedback incremental passivity and output strict incremental passivity defined for state-space systems in [7]. In general, to prove that an operator is (relaxed) co-coercive, it is possible to use a storage function approach assuming zero initial conditions (the interested reader is referred to the references [14] and [15]). Consider one of the systems in (17) and suppose that there exists a scalar function $S$ (called incremental storage function) such that for every two input-output pairs $\sigma_{1}, y_{1}$ and $\sigma_{2}, y_{2}$,

$$
\dot{S} \leq-\gamma\left\|y_{1}-y_{2}\right\|^{2}+\left(\sigma_{1}-\sigma_{2}\right)^{T}\left(y_{1}-y_{2}\right)
$$

for some $\gamma$ and every $t \geq 0$. Integrating along solutions corresponding to $x(0)=0$ and using that $S$ is nonnegative definite (so that $S(0)=0$ and $S(x(T)) \geq 0$ ), one has that

$S(x(T))-S(x(0)) \leq-\gamma\left\|y_{1}-y_{2}\right\|_{T}^{2}+\left\langle\sigma_{1}-\sigma_{2}, y_{1}-y_{2}\right\rangle_{T}$.

We conclude that $\gamma\left\|y_{1}-y_{2}\right\|_{T}^{2} \leq\left\langle\sigma_{1}-\sigma_{2}, y_{1}-y_{2}\right\rangle_{T}$, thus proving co-coercivity of the corresponding operator. 


\section{LINEAR SYSTEMS}

We specialize our results for the linear systems

$$
\begin{aligned}
\dot{x}_{k} & =A x_{k}+B v_{k} \\
y_{k} & =C x_{k},
\end{aligned} \quad k=1, \ldots, N,
$$

with arbitrary initial conditions $x_{k}^{0}$ for $k=1, \ldots, N$, and where the inputs and outputs are scalars. It is well known that for linear systems, passivity implies incremental passivity [14]. This trivially extends to operators, i.e., the non-incremental properties imply the incremental ones. A simple proof is as follows. Consider the linear operator $H$ associated to (23) (where zero initial conditions are assumed) and suppose that there exists $\gamma$, such that for every $\sigma \in L_{2 e}^{m}$ and each $T \geq 0$

$$
\langle\sigma, H \sigma\rangle_{T} \geq \gamma\|H \sigma\|_{T}^{2} .
$$

Pick $\sigma=\sigma_{1}-\sigma_{2}$. From the superposition principle we have that $H\left(\sigma_{1}-\sigma_{2}\right)=H \sigma_{1}-H \sigma_{2}$ and we conclude that

$$
\left\langle\sigma_{1}-\sigma_{2}, H \sigma_{1}-H \sigma_{2}\right\rangle_{T} \geq \gamma\left\|H \sigma_{1}-H \sigma_{2}\right\|_{T}^{2},
$$

thus proving that $H$ is relaxed co-coercive.

For asymptotically stable linear systems, $\gamma$ can be characterized (see [16] for a simple proof) as the largest $\eta$ such that

$$
\eta|G(i \omega)|^{2} \leq \operatorname{Re} G(i \omega), \quad \forall \omega \in \mathbb{R} .
$$

Expressing $G(i \omega)=n(i \omega) / q(i \omega)$ we can rewrite (24) as

$$
\eta|n(i \omega)|^{2} \leq \operatorname{Re}[n(i \omega) \overline{q(i \omega)}], \quad \forall \omega \in \mathbb{R} .
$$

We use this observation to derive the secant gain of a simple linear system (that will be useful in the next section). Consider the system $\dot{x}=-a x+b u$ with $y=x$. The transfer function is $b /(s+a)$ and therefore (24) rewrites to $\eta b^{2} \leq a b$ that leads to $\gamma=a / b$.

Clearly, to apply Theorem 2 to the systems (23) where arbitrary initial conditions are allowed, we must require that the pair $(A, B)$ is controllable.

Remark 2: In the case of $A=0, B=C=I_{n}$, system (23) with the inputs (2) becomes

$$
\dot{x}_{k}=u_{k}+\sum_{j=1}^{N} a_{k j}\left(x_{j}-x_{k}\right), \quad k=1,2, \ldots, N,
$$

that is the "classical" consensus dynamics, studied e.g., in [8], [9], perturbed with an external disturbances $u_{k} \in$ $L_{2 e}^{n}, k=1,2, \ldots, N$. Since single integrator dynamics are associated to a monotone operator with $\gamma=0$, from Theorem 2 we obtain that

$$
\|\tilde{x}\|_{T} \leq \tilde{\gamma} \hat{\beta}+\tilde{\gamma}\langle\tilde{u}, \tilde{x}\rangle_{T},
$$

where $\tilde{\gamma}=\frac{1}{\lambda}$ and $\bar{\beta}$ is a non negative constant. From (21), we conclude that

$$
\|\tilde{x}\|_{T} \leq 2 \frac{1}{\lambda} \hat{\beta}+\frac{1}{\lambda^{2}}\|\tilde{u}\|_{T}^{2},
$$

which provides a robustness condition for consensus algorithms. It is worth noting that in the case of $\tilde{u} \in L_{2}^{n(N-1)}$, (27) implies that $\tilde{x}$ is in $L_{2}^{n(N-1)}$ as well. From uniform continuity of the solutions and by using Barbalat's Lemma we conclude that $\tilde{x} \rightarrow 0$ as $t \rightarrow \infty$, thus proving consensus in the presence of $L_{2}^{n}$ perturbation signals.

\section{EXAMPLES AND APPLICATIONS}

In this section we present two scenarios that can be analyzed using the theory proposed in the present paper. The first example studies a class of networked nonlinear gradient systems, perturbed by non-identical inputs. The interest in this class of systems is motivated by the study of aggregation phenomena in biological systems like bacteria (e.g., the Escherichia coli) and in engineered applications like coordinated vehicle gradient climbing. The second example studies aggregation in networks of systems with linear dynamics with different set points.

Example 1: Consider the gradient systems

$$
\begin{aligned}
\dot{x}_{k} & =-\frac{\partial U}{\partial x_{k}}\left(x_{k}\right)+v_{k} \quad k=1, \ldots, N, \\
y_{k} & =x_{k},
\end{aligned}
$$

where $x_{k}, v_{k} \in \mathbb{R}^{n}$ and $U$ is a scalar (potential) function $U(\sigma)>0, \sigma \neq 0, U(0)=0$. Let the input be

$$
v_{k}=u_{k}+\sum_{j=1}^{n} a_{k j}\left(x_{j}-x_{k}\right), \quad k=1, \ldots, N
$$

Systems (28) can, for example, loosely describe the dynamics of a group of bacteria performing chemotaxis (where $x_{k}$ is the position of the bacteria), directing their movements according to the concentration of chemicals in their environment to find food (for example, glucose) by swimming towards the highest concentration of food molecules. The overall movement of a bacterium is the result of alternating tumble and swim phases. The tumble behavior is here modeled by the external input $u_{k} \in L_{2 e}^{n}$ in (29). The second term in (29) models the interaction amongst the bacteria, encoded by a communication graph and its associated adjacency matrix. Other possible examples include vehicle networks that must efficiently climb gradients to search and monitor where a spatially distributed environmental signal is to be mapped or its source is to be found.

We will show shortly that if the potential $U(\sigma)$ satisfies $\frac{\partial^{2} U}{\partial^{2} x_{k}} \geq \gamma I_{n}$ for some $\gamma \in \mathbb{R}$, the gradient system is relaxed co-coercive from input $v_{k}$ to the output $x_{k}$ thus satisfying one of the hypotheses of Theorem 2. For notational convenience define

$$
f\left(x_{k}\right) \triangleq \frac{\partial U}{\partial x_{k}}\left(x_{k}\right), \quad k=1,2, \ldots, N .
$$

Suppose that

$$
\frac{\partial f}{\partial x_{k}}(\sigma) \geq \gamma I_{n},
$$

for every $\sigma \in \mathbb{R}^{n}$ and some $\gamma \in \mathbb{R}$. Then each open-loop system (28) is relaxed co-coercive from the input $v_{k}$ to the output $x_{k}$. A simple proof is as follows. Let $S\left(x_{1}-x_{2}\right)=$ $1 / 2\left\|x_{1}-x_{2}\right\|^{2}$ be a candidate storage function. The time derivative is

$$
\dot{S}=\left(x_{1}-x_{2}\right)^{T}\left(\dot{x}_{1}-\dot{x}_{2}\right)
$$


Substituting (28) into (31) we obtain

$$
\dot{S}=\left(x_{1}-x_{2}\right)^{T}\left(f\left(x_{2}\right)-f\left(x_{1}\right)+v_{1}-v_{2}\right) .
$$

Defining

$$
g(\sigma)=f(\sigma)-\gamma \sigma
$$

we can rewrite (32) as

$$
\begin{aligned}
\dot{S} & =\left(x_{1}-x_{2}\right)^{T}\left(g\left(x_{2}\right)-g\left(x_{1}\right)\right) \\
& -\gamma\left\|x_{1}-x_{2}\right\|^{2}+\left(x_{1}-x_{2}\right)^{T}\left(v_{1}-v_{2}\right) .
\end{aligned}
$$

From (30) and (33) we know that the Jacobian matrix associated to the vector field $g(\cdot)$ is positive definite for every $\sigma$, i.e.,

$$
\frac{\partial g}{\partial \sigma}(\sigma) \geq 0
$$

This in turn implies that $g(\cdot)$ is a monotone (non decreasing) operator, i.e., that $\left(x_{1}-x_{2}\right)^{T}\left(g\left(x_{2}\right)-g\left(x_{1}\right)\right) \leq 0$ (see [17]). We conclude that

$$
\dot{S} \leq-\gamma\left\|x_{1}-x_{2}\right\|^{2}+\left(x_{1}-x_{2}\right)^{T}\left(v_{1}-v_{2}\right) .
$$

Integrating both sides in the interval $[0, T]$ and imposing zero initial conditions, we conclude that

$$
\gamma\left\|x_{1}-x_{2}\right\|_{T}^{2} \leq\left\langle x_{1}-x_{2}, v_{1}-v_{2}\right\rangle_{T}
$$

for each $T \geq 0$, thus proving that the operator associated to systems (28) is relaxed co-coercive with a constant $\gamma$. The second required condition on the system (i.e., reachability of the closed-loop system) is trivially satisfied. We conclude that the hypotheses of Theorem 2 are satisfied and therefore, if $\lambda>-\gamma$, there exists a $\hat{\beta} \in \mathbb{R}$ such that

$$
\|\tilde{x}\|_{T}^{2} \leq \tilde{\gamma}^{2}\|\tilde{u}\|_{T}^{2}+2 \tilde{\gamma} \hat{\beta}, \quad \forall T \geq 0
$$

Example 2: Linear systems with set points

Consider a scalar version of system (23) where $A=a<0$ and $B=C=1$. Suppose that the inputs are

$$
v_{k}=-a x_{k}^{*}+\sum_{j=1}^{n} a_{k j}\left(x_{j}-x_{k}\right), \quad k=1, \ldots, N,
$$

where $x_{k}^{*}, k=1,2, \ldots, N$ are fixed set points. The system is co-coercive with gain $\gamma=1 / a$ (as derived in Section V), and reachable. Therefore the conditions of Theorem 2 are satisfied. From (21) there exists a constant $\hat{\beta} \in \mathbb{R}$ such that the following inequality is satisfied

$$
\|\tilde{x}\|_{T}^{2} \leq 2 \hat{\beta}+\tilde{\gamma}^{2} T a^{2} \tilde{x}^{* T} \tilde{x}^{*}
$$

and we conclude that

$$
\frac{1}{T} \int_{0}^{T} \tilde{x}^{T} \tilde{x} \leq \frac{2 \hat{\beta}}{T}+a^{2} \tilde{\gamma}^{2} \tilde{x}^{* T} \tilde{x}^{*},
$$

for each $T \geq 0$. Moreover since the solutions converge to an equilibrium and (38) holds for every $T$, we can write

$$
\tilde{x}_{e q}^{T} \tilde{x}_{e q} \leq a^{2} \tilde{\gamma}^{2} \tilde{x}^{* T} \tilde{x}^{*}
$$

where $x_{e q}$ is the equilibrium vector of the system. Since $\tilde{\gamma}=1 /(\gamma+\lambda)$ we obtain the expression

$$
\tilde{x}_{e q}^{T} \tilde{x}_{e q} \leq \frac{a^{2}}{\left(a^{2}+\lambda\right)^{2}} \tilde{x}^{* T} \tilde{x}^{*} .
$$

Since the inputs are constants, we have been able to compute in closed form the bound on the output dispersion of the equilibria as a function of the dispersion of the set points. Notice that the bound decreases monotonically as $\lambda$ increases.

It is worth noting that the simple model analyzed in the present example is the linearization of the system

$\dot{\theta}_{k}=\sin \left(\theta_{k}^{*}-\theta_{k}\right)+\sum_{j=1}^{N} a_{k j} \sin \left(\theta_{j}-\theta_{k}\right), k=1,2, \ldots, N$,

where $\theta_{k}$ are heading variables for individuals moving in the plane and $\theta_{k}^{*}$ are fixed preferred directions satisfying $\left|\theta_{j}^{*}-\theta_{k}^{*}\right|<\pi / 2, k, j=1,2, \ldots, N$. Model (39) describes a biologically plausible mechanism for decision-making behavior in planar groups in motion, when there are informed subgroups with conflicting information [18].

\section{REFERENCES}

[1] M. S. Alber and A. Kiskowski, "On aggregation in CA models in biology," Journal of Physics A: Mathematical and General, vol. 34, no. 48, pp. 10707-10714, 2001.

[2] A. Stevens and F. Schweitzer, "Aggregation induced by diffusing and nondiffusing media," In W. Alt, A. Deutsch, G. Dunn (eds.), Dynamics of Cell and Tissue Motion, pp. 183-192, 1997.

[3] E. J. K. Parrish, W. M. Hamner, Animal Groups in Three Dimensions: How Species Aggregate. Cambridge University Press, 1997.

[4] P. Ogren, E. Fiorelli, and N. E. Leonard, "Cooperative control of mobile sensor networks: Adaptive gradient climbing in a distributed environment," IEEE Transactions on Automatic Control, vol. 49, no. 8, pp. 1292-1302, 2004.

[5] L. Scardovi, N. Leonard, and R. Sepulchre, "Stabilization of collective motion in three dimensions," Communications in Information and Systems, Brockett Legacy issue, 2008, to appear, preprint: arXiv: 0806.3442.

[6] R. U. Verma, "Sensitivity analysis for relaxed cocoercive nonlinear quasivariational inclusions," Journal of Applied Mathematics and Stochastic Analysis, vol. 2006, pp. 1-9, 2006.

[7] G.-B. Stan and R. Sepulchre, "Analysis of interconnected oscillators by dissipativity theory," IEEE Trans. on Automatic Control, vol. 52, no. 2, pp. 256-270, 2007.

[8] L. Moreau, "Stability of multiagent systems with time-dependent communication links," IEEE Trans. on Automatic Control, vol. 50, no. 2 , pp. 169- 182, 2005.

[9] R. Olfati-Saber and R. Murray, "Consensus problems in networks of agents with switching topology and time-delays," IEEE Trans. on Automatic Control, vol. 49, no. 9, pp. 1520- 1533, 2004.

[10] J. Wang, Y. Tan, and I. Mareels, "Robustness analysis of leaderfollower consensus," in 27th Chinese Control Conference, 2008, pp. 696-701.

[11] J. C. Willems, "Lyapunov functions for diagonally dominant systems," Automatica, vol. 12, pp. 519-523, 1976.

[12] C. W. Wu, "Algebraic connectivity of directed graphs," Linear \& Multilinear Algebra, vol. 53, no. 3, pp. 203-223, Jun 2005.

[13] J. C. Willems, The analysis of feedback systems. M.I.T. Press, 1971.

[14] R. Sepulchre, M. Jankovic, and P. V. Kokotovic, Constructive nonlinear control. Springer, 1997.

[15] A. van der Schaft, L2-Gain and Passivity Techniques in Nonlinear Control. Springer, 2000.

[16] E. D. Sontag, "Passivity gains and the "secant condition" for stability," Systems \& Control Letters, vol. 55, no. 3, pp. 177-183, 2006.

[17] F. Facchinei and J. Pang, Finite-dimensional Variational Inequalities and Complementarity Problems: Volume I. Springer, 2003.

[18] B. Nabet, N. E. Leonard, I. D. Couzin, and S. A. Levin, "Dynamics of decision-making in animal group motion," Journal of Nonlinear Science, 2009, to appear. [Online]. Available: http://www.princeton.edu/ naomi/publications 